\title{
Noniterative WLS Design of Allpass Variable Fractional-Delay Digital Filters
}

\author{
Tian-Bo Deng, Senior Member, IEEE
}

\begin{abstract}
This paper presents a noniterative weighted-leastsquares (WLS) method for designing allpass variable fractional-delay (VFD) digital filters. After expressing each coefficient of an allpass VFD filter as a polynomial of the VFD parameter $p$, we develop a noniterative technique for finding the optimal polynomial coefficients, and show that the allpass VFD filter design problem can be efficiently solved without using any iterative procedure while a closed-form solution can be easily obtained through solving a matrix equation. Compared with the existing iterative WLS method that solves a series of approximately linearized WLS minimization problems, the proposed noniterative one can yield much better design results with significantly reduced computational complexity. Moreover, the new WLS method does not involve any convergence issue.
\end{abstract}

Index Terms-Allpass variable fractional-delay (VFD) digital filter, iterative weighted-least-squares (WLS) design, noniterative WLS design, VFD digital filter, WLS design.

\section{INTRODUCTION}

$\mathbf{T}$ HE digital filters with tunable fractional phase-delay or fractional group-delay are called variable fractional-delay (VFD) filters, which are useful in various signal processing applications such as timing offset recovery in digital receivers [1], [2], comb filter design [3], [4], sampling rate conversion [5], speech coding and synthesis, time delay estimation, one-dimensional digital signal interpolation [6], and image interpolation [7], [8]. The survey paper [9] details some designs, implementations, and applications of fixed fractional-delay (FFD) filters as well as VFD filters. So far, many methods have been developed for designing and implementing finite-impulse response (FIR) VFD filters because it is relatively easy to formulate the FIR VFD filter design problem and get the optimal solutions [10]-[14]. In contrast, allpass VFD filters are much more difficult to design because the stability problem must be taken into account and the design of allpass VFD filters usually involves nonlinear optimization or iterative procedures. However, since allpass VFD filters can achieve higher design accuracy than FIR VFD filters in terms of smaller frequency response errors [15], the design and implementation of allpass VFD filters have received more attention recently [16]-[19]. Another important advantage is that allpass VFD filters have always unity magnitude response in the entire frequency band,

Manuscript received August 18, 2004; revised April 4, 2005. This paper was recommended by Associate Editor V. E. DeBrunner.

The author is with the Department of Information Science, Faculty of Science, Toho University, Chiba 274-8510, Japan (e-mail: deng @ is.sci.toho-u.ac.jp).

Digital Object Identifier 10.1109/TCSI.2005.854406 which is desired in the applications where exact unity gain is required. In [16], [17], a closed-form method has been proposed for designing and implementing maximally flat allpass VFD filters. The method is simple to use, but it is quite difficult to achieve satisfactory frequency response in the high-frequency band. To improve the design accuracy, Tseng has generalized the least-squares (LS) allpss filter design technique [20], [21] to the allpass VFD filter case [18], where the phase error is minimized through using the so-called Steiglitz-McBride iteration [22]. This iterative weighted-least-squares (WLS) method can achieve higher design accuracy than the maximally flat design even in the high-frequency band. However, since the convergence property of the Steiglitz-McBride iteration has not been uncovered yet [23], [24], it is unpredictable that whether or not the iterative WLS algorithm will converge, and to which point and in what sense the iterative algorithm will converge, our computer simulations have verified that the iterative WLS method using the Steiglitz-McBride iteration does not converge in many cases when different weighting functions are used.

This paper presents a noniterative WLS method for designing allpass VFD filters. First, each coefficient of an allpass VFD filter is expressed as a polynomial of the VFD parameter $p$. Then, the allpass VFD filter design problem is formulated as a WLS minimization problem that can be easily solved through solving a matrix equation. As a result, iterative procedures are not required in the VFD filter design process, and no convergence issue needs to be concerned. Moreover, we also exploit a new constraint that is imposed on the allpass VFD filter coefficients so that the VFD filter complexity is reduced without affecting the design accuracy. Consequently, the proposed new noniterative WLS method can achieve much better design results with significantly reduced computational complexity and less filter complexity than the existing iterative one [18].

This paper is organized as follows. Section II formulates the allpass VFD filter design as a WLS minimization problem, and derives a closed-form error function. Section III provides the optimal solution, and Section IV gives an example to illustrate the effectiveness of the proposed noniterative WLS design method. Finally, Section V concludes the paper.

\section{AllPASS VFD FILTER DESIGN}

In this section, we first formulate the allpass VFD filter design problem, then closed-form error functions are derived through using Taylor series expansions and closed-form recurrence integrals. 


\section{A. Design Problem Formulation}

The desired frequency response of an allpass VFD filter is given by

$$
H_{d}(\omega, p)=e^{-j(I+p) \omega}
$$

where $I$ is a fixed group-delay (positive integer), $p$ denotes the fractional group-delay that is continuously variable in the range

$$
\begin{aligned}
& p \in[-0.5,0.5] \\
& \omega \in[0, \alpha \pi]
\end{aligned}
$$

is the normalized angular frequency, $0<\alpha<1$. Our objective here is to find a variable allpass transfer function

$$
\begin{aligned}
H(z, p) & =\frac{a_{N}(p)+\cdots+a_{2}(p) z^{-N+2}+a_{1}(p) z^{-N+1}+z^{-N}}{1+a_{1}(p) z^{-1}+a_{2}(p) z^{-2}+\cdots+a_{N}(p) z^{-N}} \\
& =z^{-N} \cdot \frac{A\left(z^{-1}, p\right)}{A(z, p)}
\end{aligned}
$$

such that the VFD filter design specification (1) is approximated in the WLS error sense, where the denominator

$$
A(z, p)=1+\sum_{n=1}^{N} a_{n}(p) z^{-n}
$$

has the variable coefficients $a_{n}(p)$ expressed as the polynomials of the parameter $p$ as

$$
a_{n}(p)=\sum_{m=0}^{M} b_{n m} p^{m}
$$

Once the optimal coefficients $b_{n m}$ are determined, substituting different values of the parameter $p$ into (4) gets different coefficient values $a_{n}(p)$, and thus different frequency responses of $H(z, p)$. Substituting (4) into (3) yields

$$
A(z, p)=1+\sum_{n=1}^{N} \sum_{m=0}^{M} b_{n m} z^{-n} p^{m} .
$$

In the literature such as [18], the allpass VFD filter coefficients $a_{n}(p)$ are assumed to be the form (4), where for each $a_{n}(p)$, the number of coefficients $b_{n m}$ to be found is $(M+1)$. Therefore, the total number of the coefficients $b_{n m}$ in (5) is $N(M+1)$. Before proceeding to the details of the VFD filter design, let us consider the following interesting fact that can be exploited to reduce the allpass VFD filter complexity.

Rewriting (5) as

$$
A(z, p)=1+\sum_{n=1}^{N} \sum_{m=1}^{M} b_{n m} z^{-n} p^{m}+\sum_{n=1}^{N} b_{n 0} z^{-n} p^{0}
$$

and substituting $p=0$ into (6), we obtain

$$
A(z, 0)=1+\sum_{n=1}^{N} b_{n 0} z^{-n}
$$

If we let

$$
N=I
$$

and

$$
b_{n 0}=0, \quad \text { for } n=1,2, \ldots, N
$$

then

$$
\begin{aligned}
& A(z, 0)=1 \\
& H(z, 0)=z^{-N}=z^{-I}
\end{aligned}
$$

i.e.,

$$
H(\omega, 0)=e^{-j I \omega}=H_{d}(\omega, 0)
$$

Hence, imposing the constraint (7) on the VFD filter coefficients $b_{n m}$ has the following advantages.

1) The output signal $y(n)$ of the allpass VFD filter $H(z, p)$ for $p=0$ is simply the delayed version of the input signal $x(n)$, and no distortion occurs, i.e.,

$$
y(n)=x(n-N) .
$$

2) The total number of the allpass VFD filter coefficients $b_{n m}$ is reduced by $\mathrm{N}$, which in turn reduces the computational complexity of the VFD filter design and simplifies the VFD filter structure.

In this paper, we assume that the denominator $A(z, p)$ takes the form

$$
A(z, p)=1+\sum_{n=1}^{N} \sum_{m=1}^{M} b_{n m} z^{-n} p^{m}
$$

whose frequency response is

$$
\begin{aligned}
A(\omega, p) & =1+\sum_{n=1}^{N} \sum_{m=1}^{M} b_{n m} e^{-j n \omega} p^{m} \\
& =1+\sum_{n=1}^{N} \sum_{m=1}^{M} b_{n m} \cos (n \omega) p^{m}-j \sum_{n=1}^{N} \sum_{m=1}^{M} b_{n m} \sin (n \omega) p^{m} \\
& =1+\mathbf{c}^{t} \mathbf{B} \mathbf{p}-j \mathbf{s}^{t} \mathbf{B} \mathbf{p}
\end{aligned}
$$

with

$$
\begin{aligned}
& \mathbf{c}^{t}=\left[\begin{array}{llll}
\cos (\omega) & \cos (2 \omega) & \cdots & \cos (N \omega)
\end{array}\right] \\
& \mathbf{s}^{t}=\left[\begin{array}{llll}
\sin (\omega) & \sin (2 \omega) & \cdots & \sin (N \omega)
\end{array}\right] \\
& \mathbf{B}=\left[\begin{array}{cccc}
b_{11} & b_{12} & \cdots & b_{1 M} \\
b_{21} & b_{22} & \cdots & b_{2 M} \\
\vdots & \vdots & \ddots & \vdots \\
b_{N 1} & b_{N 2} & \cdots & b_{N M}
\end{array}\right] \\
& \mathbf{p}=\left[\begin{array}{c}
p \\
p^{2} \\
\vdots \\
p^{M}
\end{array}\right]
\end{aligned}
$$

It is clear that the matrix B contains all the VFD filter coefficients $b_{n m}$ that need to be determined. Based on the frequency response (9) of the denominator $A(z, p)$, we obtain the frequency response of the allpass VFD filter (2) as

$$
H(\omega, p)=e^{-j N \omega} \cdot \frac{A^{*}(\omega, p)}{A(\omega, p)}
$$

where $*$ denotes the complex conjugate. By considering the correspondence

$$
H(\omega, p) \Longleftrightarrow H_{d}(\omega, p)
$$


i.e.,

$$
e^{-j N \omega} \cdot \frac{A^{*}(\omega, p)}{A(\omega, p)} \Longleftrightarrow e^{-j(N+p) \omega}
$$

between the actual variable frequency response $H(\omega, p)$ and the desired one, we can simplify the correspondence as

$$
\frac{A^{*}(\omega, p)}{A(\omega, p)} \Longleftrightarrow \frac{e^{-j(p \omega / 2)}}{e^{j(p \omega / 2)}}
$$

which implies that we must use

$$
\frac{A^{*}(\omega, p)}{A(\omega, p)}
$$

to approximate

$$
\frac{e^{-j(p \omega / 2)}}{e^{j(p \omega / 2)}}
$$

If the above approximation is well achieved, i.e.,

$$
\frac{A^{*}(\omega, p)}{A(\omega, p)} \approx \frac{e^{-j(p \omega / 2)}}{e^{j(p \omega / 2)}}
$$

then

$$
A^{*}(\omega, p) e^{j(p \omega / 2)} \approx A(\omega, p) e^{-j(p \omega / 2)} .
$$

Using the identity

$$
A^{*}(\omega, p) e^{j(p \omega / 2)}=\left[A(\omega, p) e^{-j(p \omega / 2)}\right]^{*}
$$

the approximation in (14) can be rewritten as

$$
A(\omega, p) e^{-j(p \omega / 2)} \approx\left[A(\omega, p) e^{-j(p \omega / 2)}\right]^{*}
$$

which indicates

$$
e(\omega, p)=\operatorname{Im}\left[A(\omega, p) e^{-j(p \omega / 2)}\right] \approx 0 .
$$

Therefore, we only need to minimize the error $e(\omega, p)$. From $A(\omega, p) e^{-j(p \omega / 2)}=\left(1+\mathbf{c}^{t} \mathbf{B} \mathbf{p}-j \mathbf{s}^{t} \mathbf{B} \mathbf{p}\right)\left[\cos \left(\frac{\omega p}{2}\right)-j \sin \left(\frac{\omega p}{2}\right)\right]$ we obtain

$$
\begin{aligned}
e(\omega, p) & =\operatorname{Im}\left[A(\omega, p) e^{-j(p \omega / 2)}\right] \\
& =-\sin \left(\frac{\omega p}{2}\right)\left(1+\mathbf{c}^{t} \mathbf{B} \mathbf{p}\right)-\cos \left(\frac{\omega p}{2}\right) \mathbf{s}^{t} \mathbf{B} \mathbf{p} .
\end{aligned}
$$

Consequently, the optimal coefficient matrix $\mathbf{B}$ of the allpass VFD filter (2) can be found by minimizing the weighted squared error

$$
J(\mathbf{B})=\int_{0}^{\alpha \pi} \int_{-0.5}^{0.5} W(\omega, p) e^{2}(\omega, p) d \omega d p
$$

where $W(\omega, p)$ is a nonnegative weighting function. For deriving a closed-form error function (18), we assume that $W(\omega, p)$ is separable, i.e.,

$$
W(\omega, p)=W_{1}(\omega) W_{2}(p)
$$

and $W_{1}(\omega)$ and $W_{2}(p)$ are piecewise constant. Thus

$$
J(\mathbf{B})=\int_{0}^{\alpha \pi} \int_{-0.5}^{0.5} W_{1}(\omega) W_{2}(p) e^{2}(\omega, p) d \omega d p .
$$

From (17), we obtain

$$
\begin{aligned}
& e^{2}(\omega, p)=\sin ^{2}\left(\frac{\omega p}{2}\right)\left(1+\mathbf{c}^{t} \mathbf{B} \mathbf{p}\right)^{2}+\cos ^{2}\left(\frac{\omega p}{2}\right) \mathbf{s}^{t} \mathbf{B p p}^{t} \mathbf{B}^{t} \mathbf{s} \\
& +2 \sin \left(\frac{\omega p}{2}\right) \cos \left(\frac{\omega p}{2}\right)\left(1+\mathbf{c}^{t} \mathbf{B} \mathbf{p}\right) \mathbf{s}^{t} \mathbf{B} \mathbf{p} \\
& =\sin ^{2}\left(\frac{\omega p}{2}\right)\left(1+2 \mathbf{c}^{t} \mathbf{B} \mathbf{p}+\mathbf{c}^{t} \mathbf{B} \mathbf{p} \mathbf{p}^{t} \mathbf{B}^{t} \mathbf{c}\right) \\
& +\cos ^{2}\left(\frac{\omega p}{2}\right) \mathbf{s}^{t} \mathbf{B p p}^{t} \mathbf{B}^{t} \mathbf{s}+\sin (\omega p)\left(1+\mathbf{c}^{t} \mathbf{B} \mathbf{p}\right) \mathbf{s}^{t} \mathbf{B} \mathbf{p} \\
& =\sin ^{2}\left(\frac{\omega p}{2}\right)+2 \sin ^{2}\left(\frac{\omega p}{2}\right) \mathbf{c}^{t} \mathbf{B} \mathbf{p} \\
& +\sin ^{2}\left(\frac{\omega p}{2}\right) \mathbf{c}^{t} \mathbf{B p p}^{t} \mathbf{B}^{t} \mathbf{c} \\
& +\cos ^{2}\left(\frac{\omega p}{2}\right) \mathbf{s}^{t} \mathbf{B} \mathbf{p} \mathbf{p}^{t} \mathbf{B}^{t} \mathbf{s}+\sin (\omega p) \mathbf{s}^{t} \mathbf{B} \mathbf{p} \\
& +\sin (\omega p) \mathbf{c}^{t} \mathbf{B} \mathbf{p} \mathbf{p}^{t} \mathbf{B}^{t} \mathbf{S} \\
& =e_{1}(\omega, p)+e_{2}(\omega, p)+e_{3}(\omega, p)+e_{4}(\omega, p) \\
& +e_{5}(\omega, p)+e_{6}(\omega, p)
\end{aligned}
$$

with

$$
\begin{aligned}
& e_{1}(\omega, p)=\sin ^{2}\left(\frac{\omega p}{2}\right) \\
& e_{2}(\omega, p)=2 \sin ^{2}\left(\frac{\omega p}{2}\right) \mathbf{c}^{t} \mathbf{B} \mathbf{p} \\
& e_{3}(\omega, p)=\sin ^{2}\left(\frac{\omega p}{2}\right) \mathbf{c}^{t} \mathbf{B} \mathbf{p} \mathbf{p}^{t} \mathbf{B}^{t} \mathbf{c} \\
& e_{4}(\omega, p)=\cos ^{2}\left(\frac{\omega p}{2}\right) \mathbf{s}^{t} \mathbf{B} \mathbf{p} \mathbf{p}^{t} \mathbf{B}^{t} \mathbf{s} \\
& e_{5}(\omega, p)=\sin (\omega p) \mathbf{s}^{t} \mathbf{B} \mathbf{p} \\
& e_{6}(\omega, p)=\sin (\omega p) \mathbf{c}^{t} \mathbf{B} \mathbf{p} \mathbf{p}^{t} \mathbf{B}^{t} \mathbf{s} .
\end{aligned}
$$

Substituting the errors (22) into (20) yields

$$
J(\mathbf{B})=J_{1}+J_{2}(\mathbf{B})+J_{3}(\mathbf{B})+J_{4}(\mathbf{B})+J_{5}(\mathbf{B})+J_{6}(\mathbf{B})
$$

where

$$
\begin{aligned}
J_{1} & =\int_{0}^{\alpha \pi} \int_{-0.5}^{0.5} W_{1}(\omega) W_{2}(p) e_{1}(\omega, p) d \omega d p \\
& =\int_{0}^{\alpha \pi} \int_{-0.5}^{0.5} W_{1}(\omega) W_{2}(p) \sin ^{2}\left(\frac{\omega p}{2}\right) d \omega d p \\
& =\text { constant }
\end{aligned}
$$

is unrelated to the coefficient matrix $\mathbf{B}$, and

$$
\begin{aligned}
J_{2}(\mathbf{B}) & =\int_{0}^{\alpha \pi} \int_{-0.5}^{0.5} W_{1}(\omega) W_{2}(p) e_{2}(\omega, p) d \omega d p \\
& =\int_{0}^{\alpha \pi} \int_{-0.5}^{0.5} W_{1}(\omega) W_{2}(p) \cdot 2 \sin ^{2}\left(\frac{\omega p}{2}\right) \mathbf{c}^{t} \mathbf{B} \mathbf{p} d \omega d p \\
& =\operatorname{tr}\left[\mathbf{B} \mathbf{A}_{1}\right]
\end{aligned}
$$


with

$$
\mathbf{A}_{1}=\int_{0}^{\alpha \pi} \int_{-0.5}^{0.5} W_{1}(\omega) W_{2}(p) \cdot 2 \sin ^{2}\left(\frac{\omega p}{2}\right) \mathbf{p c}^{t} d \omega d p
$$

Similarly

$$
\begin{aligned}
J_{3}(\mathbf{B}) & =\int_{0}^{\alpha \pi} \int_{-0.5}^{0.5} W_{1}(\omega) W_{2}(p) e_{3}(\omega, p) d \omega d p \\
& =\int_{0}^{\alpha \pi} \int_{-0.5}^{0.5} W_{1}(\omega) W_{2}(p) \sin ^{2}\left(\frac{\omega p}{2}\right) \mathbf{c}^{t} \mathbf{B} \mathbf{p} \mathbf{p}^{t} \mathbf{B}^{t} \mathbf{c} d \omega d p \\
& =\operatorname{tr}\left[\mathbf{B} \int_{0}^{\alpha \pi} \int_{-0.5}^{0.5} W_{1}(\omega) W_{2}(p) \sin ^{2}\left(\frac{\omega p}{2}\right) \mathbf{p p}^{t} \mathbf{B}^{t} \mathbf{c} \mathbf{c}^{t} d \omega d p\right] \\
J_{4}(\mathbf{B}) & =\int_{0}^{\alpha \pi} \int_{-0.5}^{0.5} W_{1}(\omega) W_{2}(p) e_{4}(\omega, p) d \omega d p \\
& =\int_{0}^{\alpha \pi} \int_{-0.5}^{0.5} W_{1}(\omega) W_{2}(p) \cos ^{2}\left(\frac{\omega p}{2}\right) \mathbf{s}^{t} \mathbf{B p p}^{t} \mathbf{B}^{t} \mathbf{s} d \omega d p \\
& =\operatorname{tr}\left[\mathbf{B} \int_{0}^{\alpha \pi} \int_{-0.5}^{0.5} W_{1}(\omega) W_{2}(p) \cos ^{2}\left(\frac{\omega p}{2}\right) \mathbf{p p}^{t} \mathbf{B}^{t} \mathbf{s s} t \omega d p\right]
\end{aligned}
$$

$$
\begin{aligned}
J_{5}(\mathbf{B}) & =\int_{0}^{\alpha \pi} \int_{-0.5}^{0.5} W_{1}(\omega) W_{2}(p) e_{5}(\omega, p) d \omega d p \\
& =\int_{0}^{\alpha \pi} \int_{-0.5}^{0.5} W_{1}(\omega) W_{2}(p) \sin (\omega p) \mathbf{s}^{t} \mathbf{B} \mathbf{p} d \omega d p \\
& =\operatorname{tr}\left[\mathbf{B} \mathbf{A}_{6}\right]
\end{aligned}
$$

with

$$
\mathbf{A}_{6}=\int_{0}^{\alpha \pi} \int_{-0.5}^{0.5} W_{1}(\omega) W_{2}(p) \sin (\omega p) \mathbf{p s}^{t} d \omega d p
$$

and

$$
\begin{aligned}
J_{6}(\mathbf{B}) & =\int_{0}^{\alpha \pi} \int_{-0.5}^{0.5} W_{1}(\omega) W_{2}(p) e_{6}(\omega, p) d \omega d p \\
& =\int_{0}^{\alpha \pi} \int_{-0.5}^{0.5} W_{1}(\omega) W_{2}(p) \sin (\omega p) \mathbf{c}^{t} \mathbf{B} \mathbf{p} \mathbf{p}^{t} \mathbf{B}^{t} \mathbf{s} d \omega d p \\
& =\operatorname{tr}\left[\mathbf{B} \int_{0}^{\alpha \pi} \int_{-0.5}^{0.5} W_{1}(\omega) W_{2}(p) \sin (\omega p) \mathbf{p p}^{t} \mathbf{B}^{t} \mathbf{s} \mathbf{c}^{t} d \omega d p\right] .
\end{aligned}
$$

In the following subsections, we derive the closed-form error functions $J_{2}(\mathbf{B}), J_{3}(\mathbf{B}), \ldots, J_{6}(\mathbf{B})$.

\section{B. Closed-Form $J_{2}(\mathbf{B})$}

By using the trigonometric identity

$$
2 \sin ^{2}\left(\frac{\omega p}{2}\right)=1-\cos (\omega p)
$$

and Taylor series expansion

$$
\cos (\omega p)=1-\frac{(\omega p)^{2}}{2 !}+\frac{(\omega p)^{4}}{4 !}-\frac{(\omega p)^{6}}{6 !}+\cdots
$$

we have

$$
\begin{aligned}
2 \sin ^{2}\left(\frac{\omega p}{2}\right) & =\frac{(\omega p)^{2}}{2 !}-\frac{(\omega p)^{4}}{4 !}+\frac{(\omega p)^{6}}{6 !}-\cdots \\
& =\sum_{i=1}^{\infty} \frac{(-1)^{i-1} \cdot(\omega p)^{2 i}}{(2 i) !}
\end{aligned}
$$

Therefore, the matrix $\mathbf{A}_{1}$ in (26) can be computed as

$$
\begin{aligned}
\mathbf{A}_{1} & =\sum_{i=1}^{\infty} \frac{(-1)^{i-1}}{(2 i) !} \cdot \int_{0}^{\alpha \pi} \int_{-0.5}^{0.5} W_{1}(\omega) W_{2}(p)(\omega p)^{2 i} \mathbf{p} \mathbf{c}^{t} d \omega d p \\
& =\sum_{i=1}^{\infty} \frac{(-1)^{i-1}}{(2 i) !} \cdot \int_{-0.5}^{0.5} W_{2}(p) p^{2 i} \mathbf{p} d p \cdot \int_{0}^{\alpha \pi} W_{1}(\omega) \omega^{2 i} \mathbf{c}^{t} d \omega \\
& =\sum_{i=1}^{\infty} \mathbf{A}_{1 i}
\end{aligned}
$$

with

$$
\begin{aligned}
\mathbf{A}_{1 i} & =\frac{(-1)^{i-1}}{(2 i) !} \cdot \mathbf{u}_{i} \mathbf{v}_{i}^{t} \\
\mathbf{u}_{i} & =\int_{-0.5}^{0.5} W_{2}(p) p^{2 i} \mathbf{p} d p \\
\mathbf{v}_{i}^{t} & =\int_{0}^{\alpha \pi} W_{1}(\omega) \omega^{2 i} \mathbf{c}^{t} d \omega .
\end{aligned}
$$

Since $W_{2}(p)$ and $W_{1}(\omega)$ are assumed to be piecewise constant, the vectors $\mathbf{u}_{i}$ and $\mathbf{v}_{i}$ can be computed by using closed-form integrations. To compute the vectors $\mathbf{v}_{i}$, the recurrence formula

$$
\begin{aligned}
\int x^{m} \cos (a x) & d x=\frac{m x^{m-1} \cos (a x)}{a^{2}} \\
+ & +\frac{x^{m} \sin (a x)}{a}-\frac{m(m-1)}{a^{2}} \int x^{m-2} \cos (a x) d x
\end{aligned}
$$

is used along with

$$
\int x^{2} \cos (a x) d x=\frac{2 x \cos (a x)}{a^{2}}+\left(\frac{x^{2}}{a}-\frac{2}{a^{3}}\right) \sin (a x) .
$$

In practice, only the first several terms ( $K$ terms) in (33) need to be computed and the remaining ones can be truncated as

$$
\mathbf{A}_{1} \approx \widehat{\mathbf{A}}_{1}=\sum_{i=1}^{K} \mathbf{A}_{1 i}
$$

However, it should be noted that the matrix $\mathbf{A}_{1}$ can be approximated as accurately as desired because the series $\mathbf{A}_{1 i}$ decreases very fast in general, and thus a moderately large number $K$, say $K=9$, usually can achieve sufficiently accurate approximation, which will be illustrated later on by using computer simulations. Therefore, one needs not to worry about the truncation error caused in (34). As a result, the error function $J_{2}(\mathbf{B})$ in (25) can be approximated as

$$
J_{2}(\mathbf{B}) \approx \sum_{i=1}^{K} \operatorname{tr}\left[\mathbf{B A}_{1 i}\right]
$$

C. Closed-Form $J_{3}(\mathbf{B})$

From (32), it follows that

$$
\sin ^{2}\left(\frac{\omega p}{2}\right)=\sum_{i=1}^{\infty} \frac{(-1)^{i-1} \cdot(\omega p)^{2 i}}{2(2 i) !}
$$


Thus, $J_{3}(\mathbf{B})$ in (27) can be rewritten as (37), shown at the bottom of the page, where

$$
\begin{aligned}
\mathbf{A}_{2 i} & =\int_{-0.5}^{0.5} W_{2}(p) p^{2 i} \mathbf{p p}^{t} d p \\
& =\int_{-0.5}^{0.5} W_{2}(p) p^{2 i} \cdot\left[\begin{array}{cccc}
p^{2} & p^{3} & \cdots & p^{M+1} \\
p^{3} & p^{4} & \cdots & p^{M+2} \\
\vdots & \vdots & \ddots & \vdots \\
p^{M+1} & p^{M+2} & \cdots & p^{2 M}
\end{array}\right] d p
\end{aligned}
$$

are Hankel matrices, and the second equation at the bottom of the page are symmetric matrices. Obviously, both $\mathbf{A}_{2 i}$ and $\mathbf{A}_{3 i}$ can be computed by using closed-form integrations, and thus numerical integrals are not required. In addition, for obtaining the Hankel matrices $\mathbf{A}_{2 i}$, only their first columns and last rows need to be computed, which reduces the computational complexity. Similarly, for computing the symmetric matrices $\mathbf{A}_{3 i}$, only the elements along and below the main diagonals need to be computed. This is because the integrands $\omega^{2 i} \mathbf{c c} \mathbf{c}^{t}$ are symmetric matrices, and only the elements along and below the main diagonals are independent. Finally, the error function $J_{3}(\mathbf{B})$ can be approximated through keeping the first $K$ terms and truncating the remaining ones as

$$
J_{3}(\mathbf{B}) \approx \sum_{i=1}^{K} \operatorname{tr}\left[\mathbf{B A}_{2 i} \mathbf{B}^{t} \mathbf{A}_{3 i}\right] .
$$

In general, a moderately large number $K$ usually can get sufficiently accurate approximation, or as an extreme case, one can get almost the true result by setting a large $K$, say, $K=20$. Hence, one needs not to worry about the truncation error caused in (39). The same remarks are also applicable to the following derivations of $J_{4}(\mathbf{B}), J_{5}(\mathbf{B})$, and $J_{6}(\mathbf{B})$, and thus we do not repeat the same remarks again. Computer simulations will be given later on to verify the above remarks.

\section{Closed-Form $J_{4}(\mathbf{B})$}

It follows from (36) that

$$
\begin{aligned}
\cos ^{2}\left(\frac{\omega p}{2}\right) & =1-\sum_{i=1}^{\infty} \frac{(-1)^{i-1} \cdot(\omega p)^{2 i}}{2(2 i) !} \\
& =1+\sum_{i=2}^{\infty} \frac{(-1)^{i-1} \cdot(\omega p)^{2(i-1)}}{2(2 i-2) !} .
\end{aligned}
$$

Substituting (40) into (28) yields (41), shown at the bottom of the page, where

$$
\begin{aligned}
& \mathbf{A}_{4 i} \\
& = \begin{cases}\int_{-0.5}^{0.5} W_{2}(p) \mathbf{p} \mathbf{p}^{t} d p, & \text { if } i=1 \\
\int_{-0.5}^{0.5} W_{2}(p) p^{2(i-1)} \mathbf{p p}^{t} d p=\mathbf{A}_{2(i-1)}, & \text { if } i=2,3, \ldots\end{cases}
\end{aligned}
$$

are Hankel matrices, and

$$
\mathbf{A}_{5 i}= \begin{cases}\int_{0}^{\alpha \pi} W_{1}(\omega) \mathbf{s s}^{t} d \omega, & \text { if } i=1 \\ \frac{(-1)^{i-1}}{2(2 i-2) !} \int_{0}^{\alpha \pi} W_{1}(\omega) \omega^{2(i-1)} \mathbf{s s}^{t} d \omega, & \text { if } i=2,3, \ldots\end{cases}
$$

are symmetric matrices, where

$$
=\left[\begin{array}{cccc}
\sin ^{t}(\omega) & \sin (\omega) \sin (2 \omega) & \cdots & \sin (\omega) \sin (N \omega) \\
\sin (2 \omega) \sin (\omega) & \sin ^{2}(2 \omega) & \cdots & \sin (2 \omega) \sin (N \omega) \\
\vdots & \vdots & \ddots & \vdots \\
\sin (N \omega) \sin (\omega) & \sin (N \omega) \sin (2 \omega) & \cdots & \sin ^{2}(N \omega)
\end{array}\right] .
$$

Hence, the Hankel matrices $\mathbf{A}_{4 i}$ except $\mathbf{A}_{41}$ need not to be re-computed because $\mathbf{A}_{2 i}$ have been computed in (38), and the

$$
\begin{aligned}
J_{3}(\mathbf{B}) & =\operatorname{tr}\left[\mathbf{B} \cdot \sum_{i=1}^{\infty} \frac{(-1)^{i-1}}{2(2 i) !} \int_{-0.5}^{0.5} W_{2}(p) p^{2 i} \mathbf{p p}^{t} d p \cdot \mathbf{B}^{t} \cdot \int_{0}^{\alpha \pi} W_{1}(\omega) \omega^{2 i} \mathbf{c} \mathbf{c}^{t} d \omega\right] \\
& =\sum_{i=1}^{\infty} \operatorname{tr}\left[\mathbf{B} \mathbf{A}_{2 i} \mathbf{B}^{t} \mathbf{A}_{3 i}\right]
\end{aligned}
$$

$$
\begin{aligned}
\mathbf{A}_{3 i} & =\frac{(-1)^{i-1}}{2(2 i) !} \int_{0}^{\alpha \pi} W_{1}(\omega) \omega^{2 i} \mathbf{c c}^{t} d \omega \\
& =\frac{(-1)^{i-1}}{2(2 i) !} \int_{0}^{\alpha \pi} W_{1}(\omega) \omega^{2 i}\left[\begin{array}{cccc}
\cos ^{2}(\omega) & \cos (\omega) \cos (2 \omega) & \cdots & \cos (\omega) \cos (N \omega) \\
\cos (2 \omega) \cos (\omega) & \cos ^{2}(2 \omega) & \cdots & \cos (2 \omega) \cos (N \omega) \\
\vdots & \vdots & \ddots & \vdots \\
\cos (N \omega) \cos (\omega) & \cos (N \omega) \cos (2 \omega) & \cdots & \cos ^{2}(N \omega)
\end{array}\right] d \omega
\end{aligned}
$$

$$
\begin{aligned}
J_{4}(\mathbf{B}) & =\operatorname{tr}\left[\mathbf{B} \int_{0}^{\alpha \pi} \int_{-0.5}^{0.5} W_{2}(p) \mathbf{p} \mathbf{p}^{t} \cdot \mathbf{B}^{t} \cdot W_{1}(\omega) \mathbf{s} \mathbf{s}^{t}\left(1+\sum_{i=2}^{\infty} \frac{(-1)^{i-1} \cdot(\omega p)^{2(i-1)}}{2(2 i-2) !}\right) d \omega d p\right] \\
& =\sum_{i=1}^{\infty} \operatorname{tr}\left[\mathbf{B} \mathbf{A}_{4 i} \mathbf{B}^{t} \mathbf{A}_{5 i}\right]
\end{aligned}
$$


symmetric matrices $\mathbf{A}_{5 i}$ can be efficiently computed by using closed-form integrations. Consequently, the error function $J_{4}(\mathbf{B})$ can be truncated as

$$
J_{4}(\mathbf{B}) \approx \sum_{i=1}^{K} \operatorname{tr}\left[\mathbf{B} \mathbf{A}_{4 i} \mathbf{B}^{t} \mathbf{A}_{5 i}\right]
$$

\section{E. Closed-Form $J_{5}(\mathbf{B})$}

Substituting the Taylor series expansion

$$
\begin{aligned}
\sin (\omega p) & =(\omega p)-\frac{(\omega p)^{3}}{3 !}+\frac{(\omega p)^{5}}{5 !}-\frac{(\omega p)^{7}}{7 !}+\cdots \\
& =\sum_{i=1}^{\infty} \frac{(-1)^{i-1} \cdot(\omega p)^{2 i-1}}{(2 i-1) !}
\end{aligned}
$$

into (30) gives (45), shown at the bottom of the page, with

$$
\mathbf{A}_{6 i}=\frac{(-1)^{i-1}}{(2 i-1) !} \cdot \boldsymbol{\mu}_{i} \boldsymbol{\nu}_{i}^{t}
$$

and the vectors

$$
\begin{aligned}
& \boldsymbol{\mu}_{i}=\int_{-0.5}^{0.5} W_{2}(p) p^{2 i-1} \mathbf{p} d p \\
& \boldsymbol{\nu}_{i}^{t}=\int_{0}^{\alpha \pi} W_{1}(\omega) \omega^{2 i-1} \mathbf{s}^{t} d \omega
\end{aligned}
$$

can be computed by using closed-form integrations. To compute the vectors $\boldsymbol{\nu}_{i}$, the recurrence formula

$$
\begin{aligned}
& \int x^{m} \sin (a x) d x=\frac{m x^{m-1} \sin (a x)}{a^{2}}-\frac{x^{m} \cos (a x)}{a} \\
&-\frac{m(m-1)}{a^{2}} \int x^{m-2} \sin (a x) d x
\end{aligned}
$$

is used along with

$$
\int x \sin (a x) d x=\frac{\sin (a x)}{a^{2}}-\frac{x \cos (a x)}{a} .
$$

Finally, the matrix $\mathbf{A}_{6}$ can be truncated as

$$
\mathbf{A}_{6}=\sum_{i=1}^{\infty} \mathbf{A}_{6 i} \approx \sum_{i=1}^{K} \mathbf{A}_{6 i}
$$

and the error function $J_{5}(\mathbf{B})$ in (29) can be truncated as

$$
J_{5}(\mathbf{B}) \approx \sum_{i=1}^{K} \operatorname{tr}\left[\mathbf{B A}_{6 i}\right]
$$

\section{F. Closed-Form $J_{6}(\mathbf{B})$}

Substituting the Taylor series expansion of $\sin (\omega p)$ in (44) into (31) yields (47), shown at the bottom of the page, where

$$
\mathbf{A}_{7 i}=\int_{-0.5}^{0.5} W_{2}(p) p^{2 i-1} \mathbf{p p}^{t} d p
$$

are Hankel matrices, and (49), shown at the bottom of the next page, are asymmetric matrices. Both $\mathbf{A}_{7 i}$ and $\mathbf{A}_{8 i}$ can be computed by using closed-form integrations. Therefore, the error function $J_{6}(\mathbf{B})$ in (47) can be truncated as

$$
J_{6}(\mathbf{B}) \approx \sum_{i=1}^{K} \operatorname{tr}\left[\mathbf{B A}_{7 i} \mathbf{B}^{t} \mathbf{A}_{8 i}\right]
$$

\section{OPTIMAL SOLUTION}

Substituting (24), (35), (39), (43), (46), and (50) into (23) gives the total error function shown in the last equation at the

$$
\begin{aligned}
\mathbf{A}_{6} & =\int_{0}^{\alpha \pi} \int_{-0.5}^{0.5} W_{1}(\omega) W_{2}(p) \cdot \sum_{i=1}^{\infty} \frac{(-1)^{i-1} \cdot(\omega p)^{2 i-1}}{(2 i-1) !} \mathbf{p s}^{t} d \omega d p \\
& =\sum_{i=1}^{\infty} \frac{(-1)^{i-1}}{(2 i-1) !} \cdot\left[\int_{-0.5}^{0.5} W_{2}(p) p^{2 i-1} \mathbf{p} d p\right] \cdot\left[\int_{0}^{\alpha \pi} W_{1}(\omega) \omega^{2 i-1} \mathbf{s}^{t} d \omega\right] \\
& =\sum_{i=1}^{\infty} \mathbf{A}_{6 i}
\end{aligned}
$$

$$
\begin{aligned}
J_{6}(\mathbf{B}) & =\operatorname{tr}\left[\mathbf{B} \int_{0}^{\alpha \pi} \int_{-0.5}^{0.5} W_{1}(\omega) W_{2}(p)\left(\sum_{i=1}^{\infty} \frac{(-1)^{i-1} \cdot(\omega p)^{2 i-1}}{(2 i-1) !}\right) \mathbf{p p}^{t} \mathbf{B}^{t} \mathbf{s c}^{t} d \omega d p\right] \\
& =\sum_{i=1}^{\infty} \operatorname{tr}\left[\mathbf{B} \mathbf{A}_{7 i} \mathbf{B}^{t} \mathbf{A}_{8 i}\right]
\end{aligned}
$$


bottom of the page. The optimal coefficient matrix $\mathbf{B}$ can be determined by differentiating the error function $J(\mathbf{B})$ with respect to $\mathbf{B}$ and then setting the derivative to zero. Since

$$
\begin{aligned}
& \frac{\partial \text { constant }}{\partial \mathbf{B}}=\mathbf{0} \\
& \frac{\partial \operatorname{tr}\left[\mathbf{B} \mathbf{A}_{1 i}\right]}{\partial \mathbf{B}}=\frac{\partial \operatorname{tr}\left[\mathbf{A}_{1 i}^{t} \mathbf{B}^{t}\right]}{\partial \mathbf{B}}=\mathbf{A}_{1 i}^{t}
\end{aligned}
$$

$\frac{\partial \operatorname{tr}\left[\mathbf{B} \mathbf{A}_{2 i} \mathbf{B}^{t} \mathbf{A}_{3 i}\right]}{\partial \mathbf{B}}=\frac{\partial \operatorname{tr}\left[\mathbf{A}_{3 i}^{t} \mathbf{B} \mathbf{A}_{2 i}^{t} \mathbf{B}^{t}\right]}{\partial \mathbf{B}}=\mathbf{A}_{3 i}^{t} \mathbf{B} \mathbf{A}_{2 i}^{t}+\mathbf{A}_{3 i} \mathbf{B} \mathbf{A}_{2 i}$ $\begin{aligned} \partial \mathbf{B} & =2 \mathbf{A}_{3 i} \mathbf{B} \mathbf{A}_{2 i}\end{aligned}$

$\frac{\partial \operatorname{tr}\left[\mathbf{B A}_{4 i} \mathbf{B}^{t} \mathbf{A}_{5 i}\right]}{\partial \mathbf{B}}=\frac{\partial \operatorname{tr}\left[\mathbf{A}_{5 i}^{t} \mathbf{B} \mathbf{A}_{4 i}^{t} \mathbf{B}^{t}\right]}{\partial \mathbf{B}}=\mathbf{A}_{5 i}^{t} \mathbf{B A}_{4 i}^{t}+\mathbf{A}_{5 i} \mathbf{B} \mathbf{A}_{4 i}$ $=2 \mathbf{A}_{5 i} \mathbf{B A}_{4 i}$

$\frac{\partial \operatorname{tr}\left[\mathbf{B} \mathbf{A}_{6 i}\right]}{\partial \mathbf{B}}=\frac{\partial \operatorname{tr}\left[\mathbf{A}_{6 i}^{t} \mathbf{B}^{t}\right]}{\partial \mathbf{B}}=\mathbf{A}_{6 i}^{t}$

$\frac{\partial \operatorname{tr}\left[\mathbf{B} \mathbf{A}_{7 i} \mathbf{B}^{t} \mathbf{A}_{8 i}\right]}{\partial \mathbf{B}}=\frac{\partial \operatorname{tr}\left[\mathbf{A}_{8 i}^{t} \mathbf{B} \mathbf{A}_{7 i}^{t} \mathbf{B}^{t}\right]}{\partial \mathbf{B}}=\mathbf{A}_{8 i}^{t} \mathbf{B} \mathbf{A}_{7 i}^{t}+\mathbf{A}_{8 i} \mathbf{B} \mathbf{A}_{7 i}$

$$
=\left(\mathbf{A}_{8 i}^{t}+\mathbf{A}_{8 i}\right) \mathbf{B} \mathbf{A}_{7 i}=2 \mathbf{A}_{9 i} \mathbf{B} \mathbf{A}_{7 i}
$$

with

$$
\mathbf{A}_{9 i}=\frac{\mathbf{A}_{8 i}^{t}+\mathbf{A}_{8 i}}{2}
$$

thus

$$
\begin{aligned}
\frac{\partial J(\mathbf{B})}{\partial \mathbf{B}} & =\sum_{i=1}^{K}\left[\mathbf{A}_{1 i}^{t}+2 \mathbf{A}_{3 i} \mathbf{B A}_{2 i}+2 \mathbf{A}_{5 i} \mathbf{B A}_{4 i}+\mathbf{A}_{6 i}^{t}+2 \mathbf{A}_{9 i} \mathbf{B A}_{7 i}\right] \\
& =\mathbf{0}
\end{aligned}
$$

i.e.,

$$
\begin{aligned}
\sum_{i=1}^{K}\left[\mathbf{A}_{3 i} \mathbf{B A}_{2 i}+\mathbf{A}_{5 i} \mathbf{B} \mathbf{A}_{4 i}+\right. & \left.\mathbf{A}_{9 i} \mathbf{B} \mathbf{A}_{7 i}\right] \\
& =-\sum_{i=1}^{K}\left(\frac{\mathbf{A}_{1 i}^{t}+\mathbf{A}_{6 i}^{t}}{2}\right) .
\end{aligned}
$$

Applying the identity

$$
\operatorname{cs}(\mathbf{A X C})=\left(\mathbf{C}^{t} \otimes \mathbf{A}\right) \operatorname{cs}(\mathbf{X})
$$

to $(51)$, where $\operatorname{cs}(\mathbf{X})$ represents the column string of the matrix $\mathbf{X}$, and $\otimes$ denotes the Kronecker product, then

$$
\begin{aligned}
\sum_{i=1}^{K}\left[\mathbf{A}_{2 i}^{t} \otimes \mathbf{A}_{3 i}+\mathbf{A}_{4 i}^{t} \otimes \mathbf{A}_{5 i}+\right. & \left.\mathbf{A}_{7 i}^{t} \otimes \mathbf{A}_{9 i}\right] \operatorname{cs}(\mathbf{B}) \\
& =-\operatorname{cs}\left[\sum_{i=1}^{K}\left(\frac{\mathbf{A}_{1 i}^{t}+\mathbf{A}_{6 i}^{t}}{2}\right)\right] .
\end{aligned}
$$

Because the matrices $\mathbf{A}_{2 i}, \mathbf{A}_{4 i}$, and $\mathbf{A}_{7 i}$ are symmetric, thus

$$
\begin{aligned}
& \sum_{i=1}^{K}\left[\mathbf{A}_{2 i} \otimes \mathbf{A}_{3 i}+\mathbf{A}_{4 i} \otimes \mathbf{A}_{5 i}+\mathbf{A}_{7 i} \otimes \mathbf{A}_{9 i}\right] \operatorname{cs}(\mathbf{B}) \\
&=-\operatorname{cs}\left[\sum_{i=1}^{K}\left(\frac{\mathbf{A}_{1 i}^{t}+\mathbf{A}_{6 i}^{t}}{2}\right)\right] .
\end{aligned}
$$

Since the matrix $\mathbf{B}$ here actually represents the VFD filter coefficients that minimize (20), although quite large size of $\mathbf{B}$ may lead to ill-conditioned problem, the optimal solution for such a practical minimization problem with a reasonably large size of $\mathbf{B}$ always exists. Solving the matrix (52) yields the optimal column vector $\operatorname{cs}(\mathbf{B})$, and thus the optimal coefficient matrix $\mathbf{B}$ can be obtained from $\operatorname{cs}(\mathbf{B})$.

Before concluding this section, we provide some comments on the proposed noniterative WLS design method. First, let us see what is the relation between the error function $e(\omega, p)$ defined in (16) and the frequency response error

$$
\begin{aligned}
e_{H}(\omega, p) & =H(\omega, p)-H_{d}(\omega, p) \\
& =e^{-j N \omega} \cdot \frac{A^{*}(\omega, p)}{A(\omega, p)}-e^{-j(N+p) \omega} \\
& =e^{-j N \omega}\left[\frac{A^{*}(\omega, p)}{A(\omega, p)}-e^{-j p \omega}\right] .
\end{aligned}
$$

$$
\begin{aligned}
\mathbf{A}_{8 i} & =\frac{(-1)^{i-1}}{(2 i-1) !} \int_{0}^{\alpha \pi} W_{1}(\omega) \omega^{2 i-1} \cdot \mathbf{s c}^{t} d \omega \\
& =\frac{(-1)^{i-1}}{(2 i-1) !} \int_{0}^{\alpha \pi} W_{1}(\omega) \omega^{2 i-1} \cdot\left[\begin{array}{c}
\sin (\omega) \\
\sin (2 \omega) \\
\vdots \\
\sin (N \omega)
\end{array}\right]\left[\begin{array}{cccc}
\cos (\omega) & \cos (2 \omega) & \cdots & \cos (N \omega)] d \omega \\
\sin (\omega) \cos (\omega) & \sin (\omega) \cos (2 \omega) & \cdots & \sin (\omega) \cos (N \omega) \\
\sin (2 \omega) \cos (\omega) & \sin (2 \omega) \cos (2 \omega) & \cdots & \sin (2 \omega) \cos (N \omega) \\
\vdots & \vdots & \ddots & \vdots \\
\sin (N \omega) \cos (\omega) & \sin (N \omega) \cos (2 \omega) & \cdots & \sin (N \omega) \cos (N \omega)
\end{array}\right] d \omega
\end{aligned}
$$

$$
J(\mathbf{B})=\text { constant }+\sum_{i=1}^{K}\left(\operatorname{tr}\left[\mathbf{B} \mathbf{A}_{1 i}\right]+\operatorname{tr}\left[\mathbf{B} \mathbf{A}_{2 i} \mathbf{B}^{t} \mathbf{A}_{3 i}\right]+\operatorname{tr}\left[\mathbf{B} \mathbf{A}_{4 i} \mathbf{B}^{t} \mathbf{A}_{5 i}\right]+\operatorname{tr}\left[\mathbf{B} \mathbf{A}_{6 i}\right]+\operatorname{tr}\left[\mathbf{B} \mathbf{A}_{7 i} \mathbf{B}^{t} \mathbf{A}_{8 i}\right]\right)
$$


TABLE I

TRUNCATION ERRORS

\begin{tabular}{c|c|c|c|c|c}
\hline $\mathrm{K}$ & $\epsilon_{J_{2}}(\%)$ & $\epsilon_{J_{3}}(\%)$ & $\epsilon_{J_{4}}(\%)$ & $\epsilon_{J_{5}}(\%)$ & $\epsilon_{J_{6}}(\%)$ \\
\hline \hline 1 & 8.682347930479 & 9.177906330279 & 20.232226720672 & 12.857690231358 & 16.918632411004 \\
\hline 2 & 0.340337926475 & 0.411428775810 & 1.852739617549 & 0.673045189453 & 0.756070581650 \\
\hline 3 & 0.007849085343 & 0.010717779353 & 0.078105291487 & 0.019679574148 & 0.015292061092 \\
\hline 4 & 0.000120523857 & 0.000182342386 & 0.001930225663 & 0.000369781391 & 0.000146614158 \\
\hline 5 & 0.000001326898 & 0.000002188164 & 0.000031541644 & 0.000004852304 & 0.000000119779 \\
\hline 6 & 0.000000011012 & 0.000000019540 & 0.000000367038 & 0.000000047026 & 0.000000015206 \\
\hline 7 & 0.000000000071 & 0.000000000135 & 0.000000003200 & 0.000000000350 & 0.000000000225 \\
\hline 8 & 0.000000000000 & 0.000000000000 & 0.000000000021 & 0.000000000002 & 0.000000000001 \\
\hline 9 & 0.000000000000 & 0.000000000000 & 0.000000000000 & 0.000000000000 & 0.000000000000 \\
\hline$\vdots$ & $\vdots$ & $\vdots$ & $\vdots$ & $\vdots$ & $\vdots$ \\
\hline
\end{tabular}

Since $e_{H}(\omega, p)$ can be manipulated to

$$
\begin{aligned}
e_{H}(\omega, p) & =\frac{e^{-j N \omega}\left[A^{*}(\omega, p)-A(\omega, p) e^{-j p \omega}\right]}{A(\omega, p)} \\
& =\frac{e^{-j(N+(p / 2)) \omega}\left[A^{*}(\omega, p) e^{j(p / 2) \omega}-A(\omega, p) e^{-j(p / 2) \omega}\right]}{A(\omega, p)} \\
& =\frac{e^{-j(N+(p / 2)) \omega} \cdot 2 \operatorname{Im}\left[A^{*}(\omega, p) e^{j(p / 2) \omega}\right]}{A(\omega, p)} \\
& =-\frac{e^{-j(N+(p / 2)) \omega} \cdot 2 \operatorname{Im}\left[A(\omega, p) e^{-j(p / 2) \omega}\right]}{A(\omega, p)} \\
& =-\frac{e^{-j(N+(p / 2)) \omega} \cdot 2 e(\omega, p)}{A(\omega, p)}
\end{aligned}
$$

thus,

$$
\left|e_{H}(\omega, p)\right|=\frac{2|e(\omega, p)|}{|A(\omega, p)|} .
$$

Next, let us consider the WLS design that minimizes the weighted squared error of variable frequency response

$$
\begin{aligned}
J_{H}(\mathbf{B}) & =\int_{0}^{\alpha \pi} \int_{-0.5}^{0.5} W(\omega, p)\left|e_{H}(\omega, p)\right|^{2} d \omega d p \\
& =\int_{0}^{\alpha \pi} \int_{-0.5}^{0.5} W(\omega, p) \frac{4 e^{2}(\omega, p)}{|A(\omega, p)|^{2}} d \omega d p .
\end{aligned}
$$

By comparing (54) with (18), we know that the proposed noniterative WLS design is equivalent to the WLS design that minimizes $J_{H}(\mathbf{B})$ with $|A(\omega, p)|$ fixed. In [18], $|A(\omega, p)|$ is regarded as part of the weighting function, and then the Steiglitz-McBride iteration is used to minimize the linearized phase error function. Although the final solutions from both the iterative WLS design [18] and the proposed noniterative one can only be claimed to be "locally optimal" (sub-optimal), our computer simulations have demonstrated that the proposed noniterative WLS technique can yield much better design results with significantly reduced computational complexity and less VFD filter complexity than the iterative one [18]. Furthermore, the convergence issue needs not to be concerned here.

\section{DESIGn EXAMPLe}

This section presents an example to illustrate the effectiveness of the proposed noniterative WLS technique and compare it with the existing iterative one [18].

\section{A. Example}

The variable design specification (1) is approximated in the region

$$
\begin{aligned}
& \omega \in[0, \alpha \pi], \quad \alpha=0.9 \\
& p \in[-0.5,0.5]
\end{aligned}
$$

with the same allpass VFD filter order and polynomial degree as [18]

$$
\begin{aligned}
& N=35 \\
& M=5 .
\end{aligned}
$$

To perform the WLS design using the method [18], the frequency $\omega$ and fractional-delay $p$ are uniformly sampled as

$$
\begin{aligned}
& \omega\left(l_{1}\right)=\frac{\alpha \pi l_{1}}{L_{1}}, \quad l_{1}=0,1,2, \ldots L_{1} \\
& p\left(l_{2}\right)=-0.5+\frac{l_{2}}{L_{2}}, \quad l_{2}=0,1,2, \ldots L_{2}
\end{aligned}
$$

with

$$
\begin{aligned}
& L_{1}=100 \\
& L_{2}=30 .
\end{aligned}
$$

Note that increasing the numbers $L_{1}$ and $L_{2}$ may improve the design accuracy with increased computational complexity in the WLS design [18], but there is not such a tradeoff with the proposed noniterative approach.

To perform the WLS design using the noniterative method, we must determine the number $K$ in (52) first. Table I and Fig. 1 show the the normalized root-mean-squared (RMS) truncation errors of $J_{2}(\mathbf{B}), J_{3}(\mathbf{B}), J_{4}(\mathbf{B}), J_{5}(\mathbf{B})$, and $J_{6}(\mathbf{B})$ defined by

$$
\epsilon_{J_{i}}=\frac{\left|J_{i}(\mathbf{B})-\widehat{J}_{i}(\mathbf{B})\right|}{\left|J_{i}(\mathbf{B})\right|} \times 100 \%, \quad i=2,3,4,5,6
$$




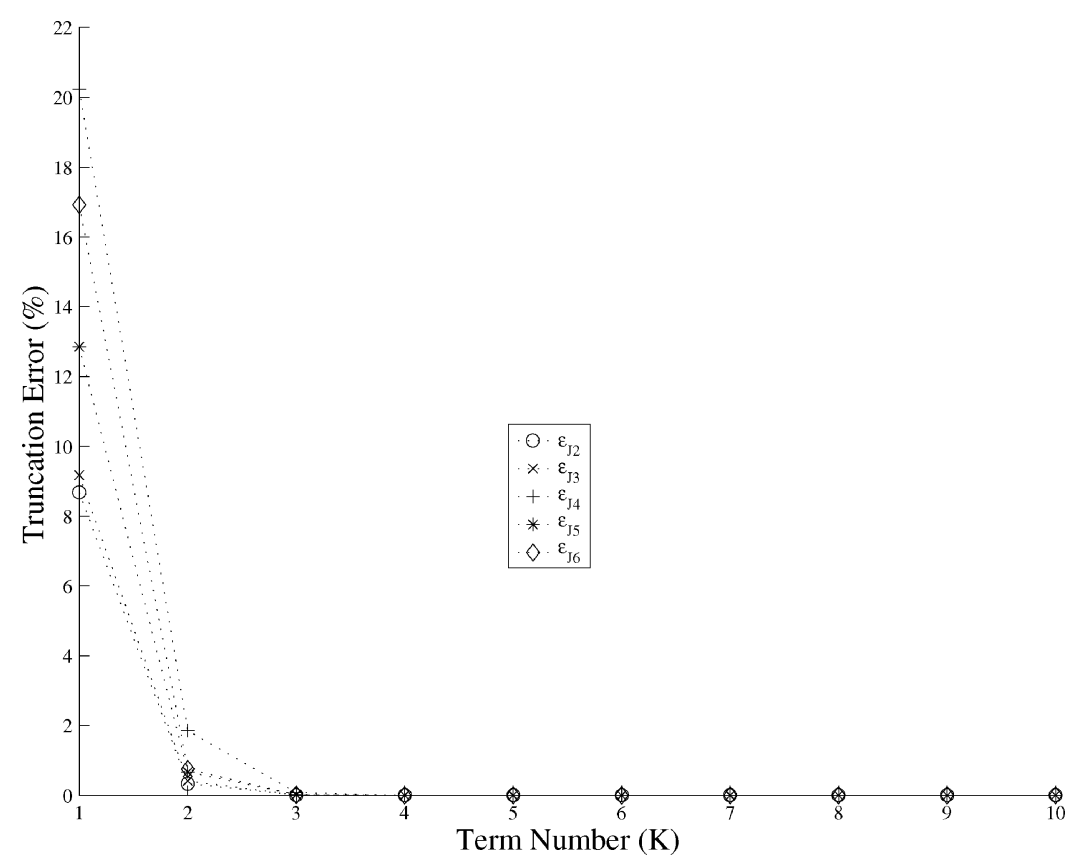

Fig. 1. Truncation errors $\epsilon_{J_{i}}$ for different $K$.

where $J_{i}(\mathbf{B})$ are the ideal values computed by using a very large $K(K=20)$, and $\widehat{J}_{i}(\mathbf{B})$ are the actual values for different $K$. Also, the weighting functions are set as

$$
\begin{array}{ll}
W_{1}(\omega)=1, & \text { for } \omega \in[0, \alpha \pi] \\
W_{2}(p)=1, & \text { for } p \in[-0.5,0.5] .
\end{array}
$$

From Table I and Fig. 1 it is observed that the truncation errors $\epsilon_{J_{i}}$ decrease very fast. For example, if we set $K=9$, then all the truncation errors approach zero. Therefore, we set $K=9$ in the practical designs.

To evaluate the VFD filter design accuracy, the normalized RMS error $\epsilon_{2}$ of the variable frequency response and maximum frequency response error $\epsilon_{\mathrm{Max}}$ defined by

$$
\begin{aligned}
& \epsilon_{2}=\left[\frac{\int_{0}^{\alpha \pi} \int_{-0.5}^{0.5}\left|e_{H}(\omega, p)\right|^{2} d \omega d p}{\int_{0}^{\alpha \pi} \int_{-0.5}^{0.5}\left|H_{d}(\omega, p)\right|^{2} d \omega d p}\right]^{1 / 2} \times 100 \% \\
& \epsilon_{\operatorname{Max}} \\
& \quad=\max \left\{20 \log _{10}\left|e_{H}(\omega, p)\right|, \omega \in[0, \alpha \pi], p \in[-0.5,0.5]\right\}
\end{aligned}
$$

the normalized RMS fractional-delay error $\epsilon_{p 2}$ and maximum fractional-delay error $\epsilon_{p \text { Max }}$ defined by

$$
\begin{aligned}
\epsilon_{p 2} & =\left[\frac{\int_{0}^{\alpha \pi} \int_{-0.5}^{0.5}\left|e_{p}(\omega, p)\right|^{2} d \omega d p}{\int_{0}^{\alpha \pi} \int_{-0.5}^{0.5} p^{2} d \omega d p}\right]^{1 / 2} \times 100 \% \\
\epsilon_{p \operatorname{Max}} & =\max \left\{\left|e_{p}(\omega, p)\right|, \omega \in[0, \alpha \pi], p \in[-0.5,0.5]\right\}
\end{aligned}
$$

and the normalized RMS phase error $\epsilon_{\theta 2}$ and maximum phase error $\epsilon_{\theta \mathrm{Max}}$ defined by

$$
\begin{aligned}
\epsilon_{\theta 2} & =\left[\frac{\int_{0}^{\alpha \pi} \int_{-0.5}^{0.5}\left|e_{\theta}(\omega, p)\right|^{2} d \omega d p}{\int_{0}^{\alpha \pi} \int_{-0.5}^{0.5} \theta_{d}^{2}(\omega, p) d \omega d p}\right]^{1 / 2} \times 100 \% \\
\epsilon_{\theta \operatorname{Max}} & =\max \left\{\left|e_{\theta}(\omega, p)\right|, \omega \in[0, \alpha \pi], p \in[-0.5,0.5]\right\}
\end{aligned}
$$

are used. In (58), the frequency response error $e_{H}(\omega, p)$ is defined in (53). In (59),

$$
e_{p}(\omega, p)=\tau(\omega, p)-p
$$

is the fractional group-delay error, $\tau(\omega, p)$ and $p$ are the actual and desired fractional group-delays, respectively. In (60),

$$
e_{\theta}(\omega, p)=\theta(\omega, p)-\theta_{d}(\omega, p)
$$

is the phase error, $\theta(\omega, p)$ and $\theta_{d}(\omega, p)$ are the actual and ideal phase responses, respectively. From (1), it follows that

$$
\theta_{d}(\omega, p)=-(I+p) \omega .
$$

To evaluate the above six types of design errors, the discrete points in (56) are set as

$$
\begin{aligned}
& L_{1}=200 \\
& L_{2}=60 .
\end{aligned}
$$

Our computer simulations are performed on a Dell PC with Intel Pentium4-2 GHz CPU. At first, let us check the normal (pure) least-squares (LS) design results, i.e., the weighting functions are set as (57). To perform the LS design using the iterative WLS method [18], the initial filter coefficients are set to zero, i.e., the initial denominator $A(z, p)$ in (2) is

$$
A(z, p)=1 \text {. }
$$

The iterative algorithm converges to a local minimum after 3 iterations when the terminating criterion $(\xi)$ defined in [18] is set to $10^{-4}$. Table II lists the design errors of the proposed noniterative WLS method and the iterative one along with the CPU time and the numbers of VFD filter coefficients. Table II indicates that the proposed noniterative WLS method can achieve higher design accuracy with significantly reduced CPU time than the iterative one [18]. Moreover, the new WLS method also requires fewer filter coefficients than the iterative one because the new coefficient constraint (7) is imposed on the allpass VFD filter design. 
TABLE II

DESIGN ERRors AND ALLPASS VFD FILTER COMPLEXITIES

\begin{tabular}{c|c|c|c|c|c|c|c|c}
\hline Method & $\epsilon_{2}(\%)$ & $\epsilon_{\operatorname{Max}}(\mathrm{dB})$ & $\epsilon_{p 2}(\%)$ & $\epsilon_{p \operatorname{Max}}$ & $\epsilon_{\theta 2}(\%)$ & $\epsilon_{\theta \operatorname{Max}}$ & CPU (sec.) & Coefficients \\
\hline \hline $\begin{array}{c}\text { Non-iterative } \\
\text { LS } \\
\text { Design }\end{array}$ & 0.000562 & -74.95 & 0.1260 & 0.0158 & 0.0012 & 0.000179 & 1.87 & 175 \\
\hline $\begin{array}{c}\text { Iterative } \\
\text { LS } \\
\text { Design [18] }\end{array}$ & 0.001146 & -65.14 & 0.2693 & 0.0369 & 0.0024 & 0.000553 & 63.21 & 210 \\
\hline $\begin{array}{c}\text { Non-iterative } \\
\text { WLS } \\
\text { Design }\end{array}$ & 0.000654 & -86.43 & 0.0994 & 0.0067 & 0.0014 & 0.000048 & 3.41 & 175 \\
\hline $\begin{array}{c}\text { Iterative } \\
\text { WLS } \\
\text { Design [18] }\end{array}$ & 0.000996 & -67.49 & 0.2294 & 0.0291 & 0.0021 & 0.000422 & 63.27 & 210 \\
\hline
\end{tabular}

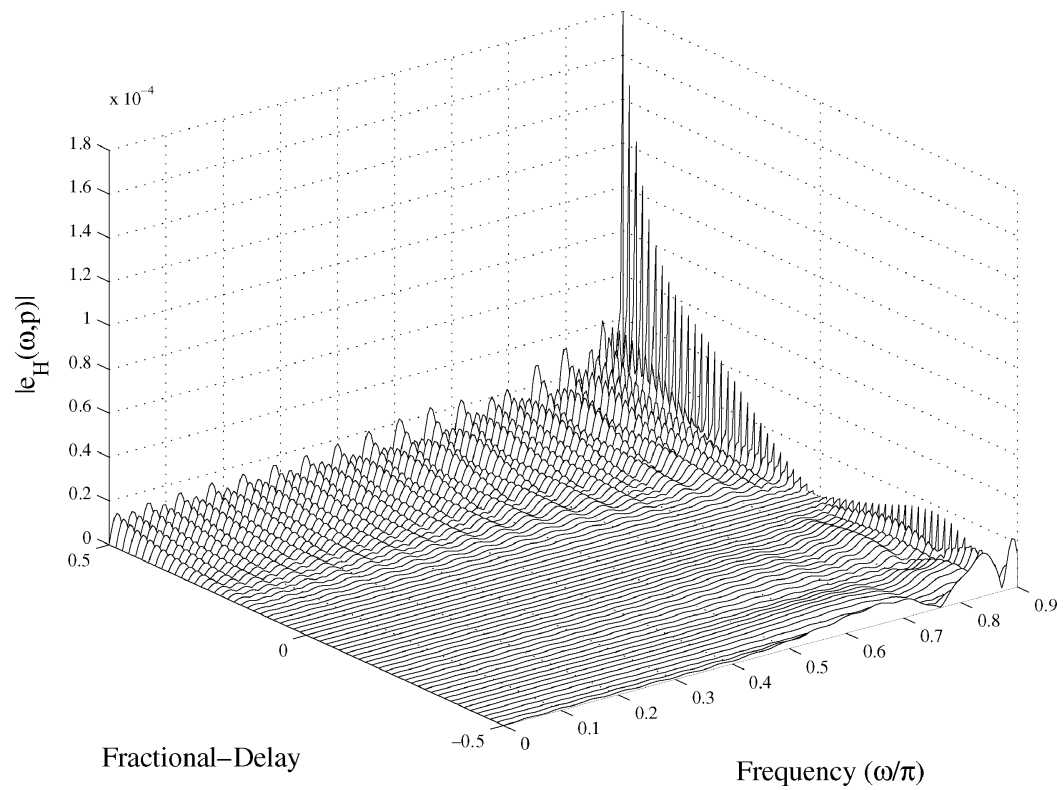

Fig. 2. Absolute error of variable frequency response (noniterative LS design).

Fig. 2 shows the absolute error $\left|e_{H}(\omega, p)\right|$ of the variable frequency response from the proposed LS design, and Fig. 3 depicts the maximum radius of the poles of the allpass VFD filter as the value of the fractional-delay $p$ varies. Because all the poles are inside the unit circle, the resulting allpass VFD filter is stable. As discussed in [18], although there is no theoretical guarantee for the stability of the resulting allpass VFD filter, if the actual variable frequency response approximates the desired one accurately, which implies the actual and desired phase responses match well, the designed allpass VFD filter is usually stable. Fig. 2 also clearly shows that the frequency response errors are not flat. To suppress the peak errors and make the error distribution as flat as possible, the following weighting functions:

$$
\begin{aligned}
& W_{1}(\omega)= \begin{cases}0.2703, & \omega \in[0,0.855 \pi) \\
1, & \omega \in[0.855 \pi,(\alpha+\delta) \pi], \quad \delta=0.00124\end{cases} \\
& W_{2}(p)= \begin{cases}0.0633, & p \in[-0.5,0.4) \\
1, & p \in[0.4,0.5)\end{cases}
\end{aligned}
$$

are used, where the small number $\delta$ is added for suppressing the error jumps around the edge frequency $\omega=\alpha \pi$. Table II lists the design results from the two WLS methods, which show that the proposed noniterative method still can achieve much better results with significantly reduced computational cost than the iterative one. Also, since the weighting functions here are used for suppressing the peak errors, it can be seen from Table II that the maximum errors ( $\epsilon_{\mathrm{Max}}, \epsilon_{p \operatorname{Max}}$, and $\left.\epsilon_{\theta \mathrm{Max}}\right)$ are reduced at the sacrifice of slightly increased RMS errors. If one seeks to minimize the total squared error of variable frequency response, then the normal (pure) LS design (WLS design without weightings) is preferred.

Our computer simulations using various weighting functions have also indicated that the iterative WLS method has severe convergence problem. In many cases, it does not converge. This is due to the fact that the convergence property of the so-called Steiglitz-McBride iteration used in [18] is still unknown at this point, and it may not converge especially for nonconstant weighting functions [22]-[24]. 


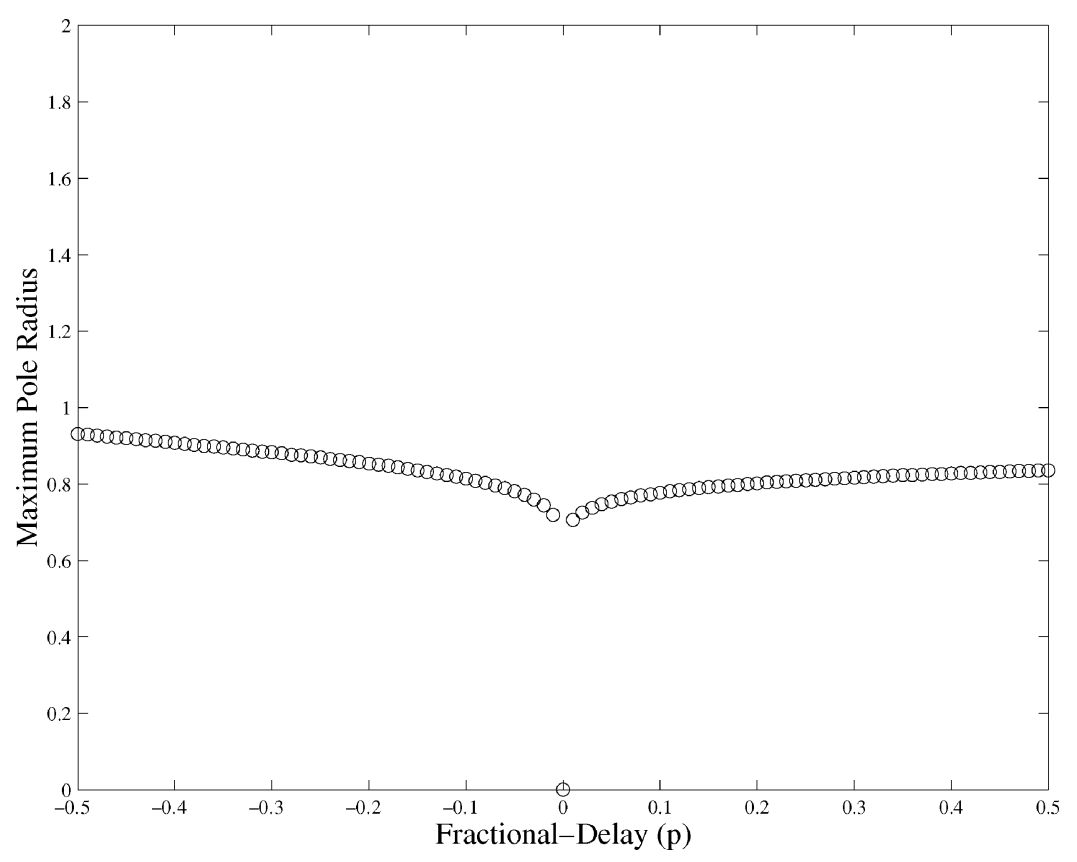

Fig. 3. Maximum pole radius (noniterative LS design).

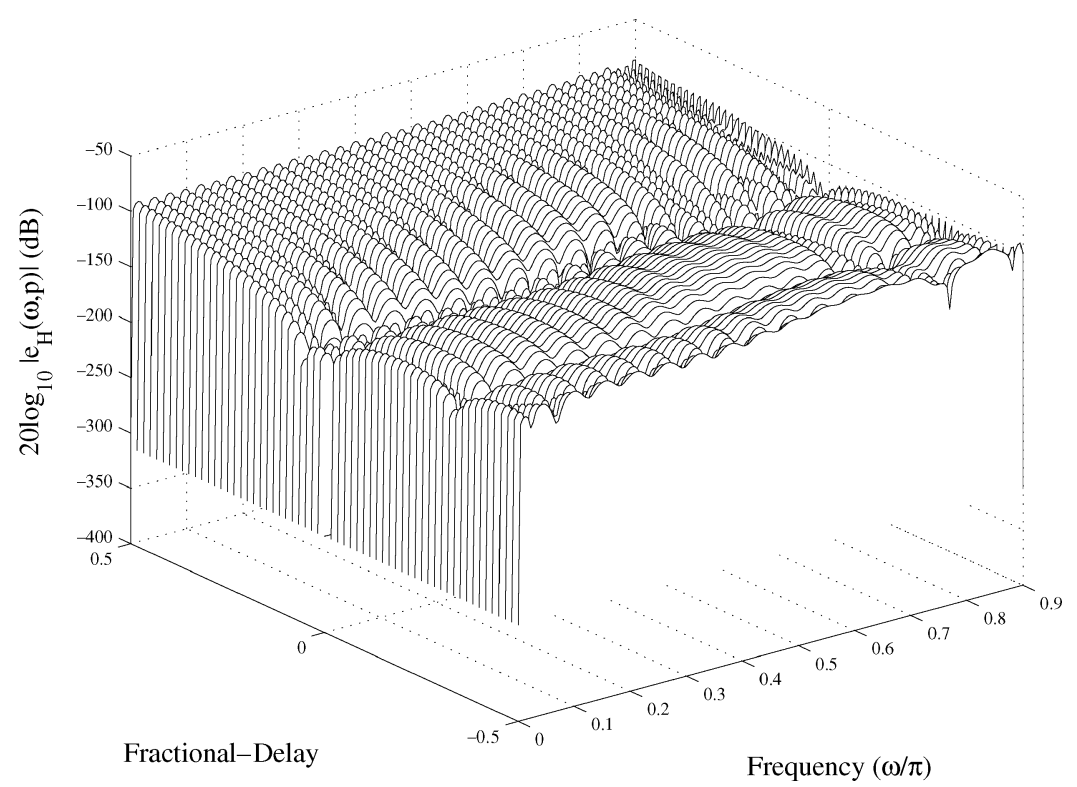

Fig. 4. Variable frequency response error (noniterative WLS design).

To illustrate the results from the proposed noniterative WLS approach, Fig. 4 shows the frequency response error in dB, Figs. 5 and 6 plot the actual variable fractional group-delay and its absolute error. To check the stability, Fig. 7 depicts the maximum radius of the poles when varying the fractional-delay $p$. Obviously, all the poles are inside the unit circle, thus the resulting allpass VFD filter is stable.

In [19], a minimax method has been proposed for designing an allpass VFD filter with minimum phase-delay error. Generally speaking, it is not fair to compare a WLS design with a minimax design because the objective of the minimax design is to minimize the worst-case (maximum) error, while the objective of the WLS design is basically to minimize the total error energy. In some applications, the WLS design is more important than the minimax design because the total error energy from the WLS design is usually much smaller than that from the minimax design. On the other hand, if one wants to know the worst-case error, then the minimax design is preferred. However, it should be mentioned here that the design result from our noniterative WLS method can be chosen as a good starting point (initial guess) for other designs such as minimax design. For example, assume that the same design specification in [19] is to be approximated such that the maximum phase-delay error is minimized. If we use the VFD filter coefficients from the noniterative WLS design as initial values, and the minimax design is carried out using the Matlab function fminimax, then we can reach a sub-optimal solution in 2 iterations, it takes only 3.5 seconds. The maximum phase-delay error is 0.00879 , which is slightly 


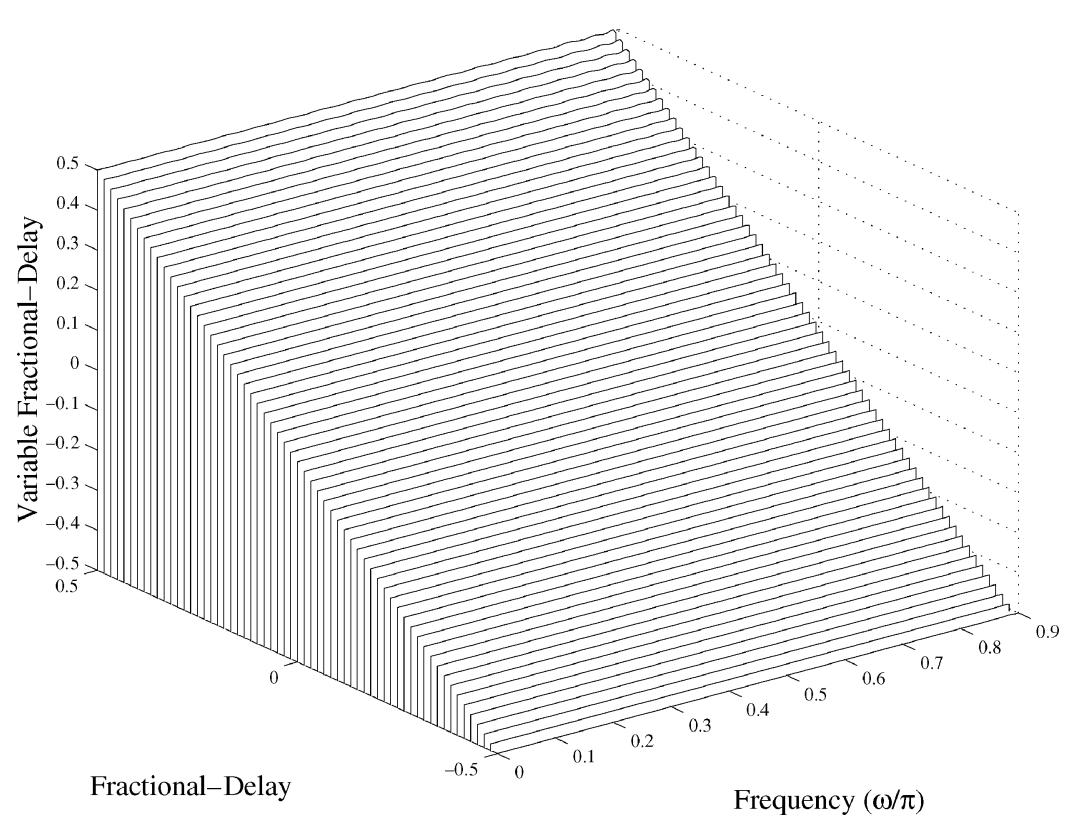

Fig. 5. VFD (noniterative WLS design).

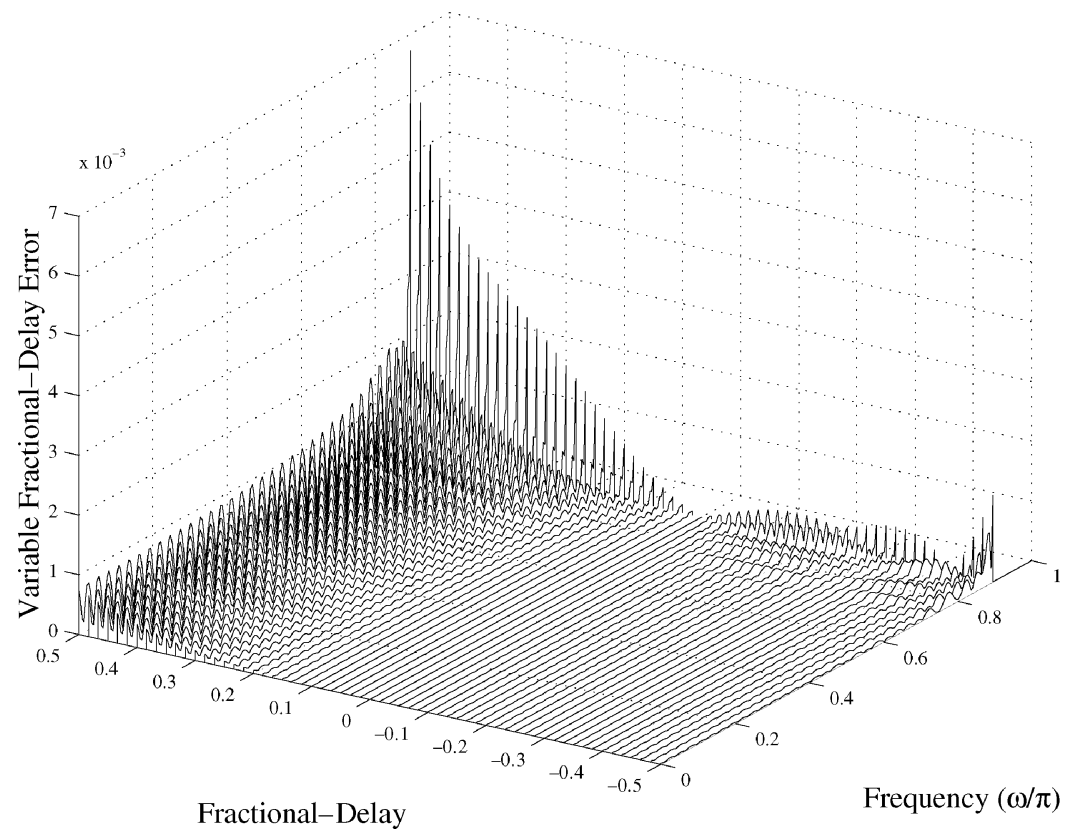

Fig. 6. VFD error (noniterative WLS design).

smaller than that (0.008 94) in [19], and the maximum radius of the poles is 0.99440 .

\section{CONCLUSION}

This paper has proposed a noniterative WLS technique for designing allpass VFD digital filters. Compared with the existing iterative one that utilizes the Steiglitz-McBride iteration [18], the proposed noniterative WLS method has the following advantages.

1) The WLS design can be rigorously derived without using parameter discretizations and numerical integrals. It is obtained through using Taylor series expansions and the corresponding closed-form integrals.
2) A new coefficient constraint is exploited in the WLS design so that the allpass VFD filter complexity in terms of the total number of VFD filter coefficients is reduced. The new constraint also guarantees no signal distortion for $p=0$. Our computer simulations have verified that the imposed constraint only causes neglectable increased design errors as compared to the WLS design without imposing the constraint. Therefore, imposing the coefficient constraint makes it possible to achieve comparable design accuracy with reduced VFD filter complexity.

3) The noniterative WLS design does not involve any convergence issue, which obtains the sub-optimal solution through solving a matrix equation. 


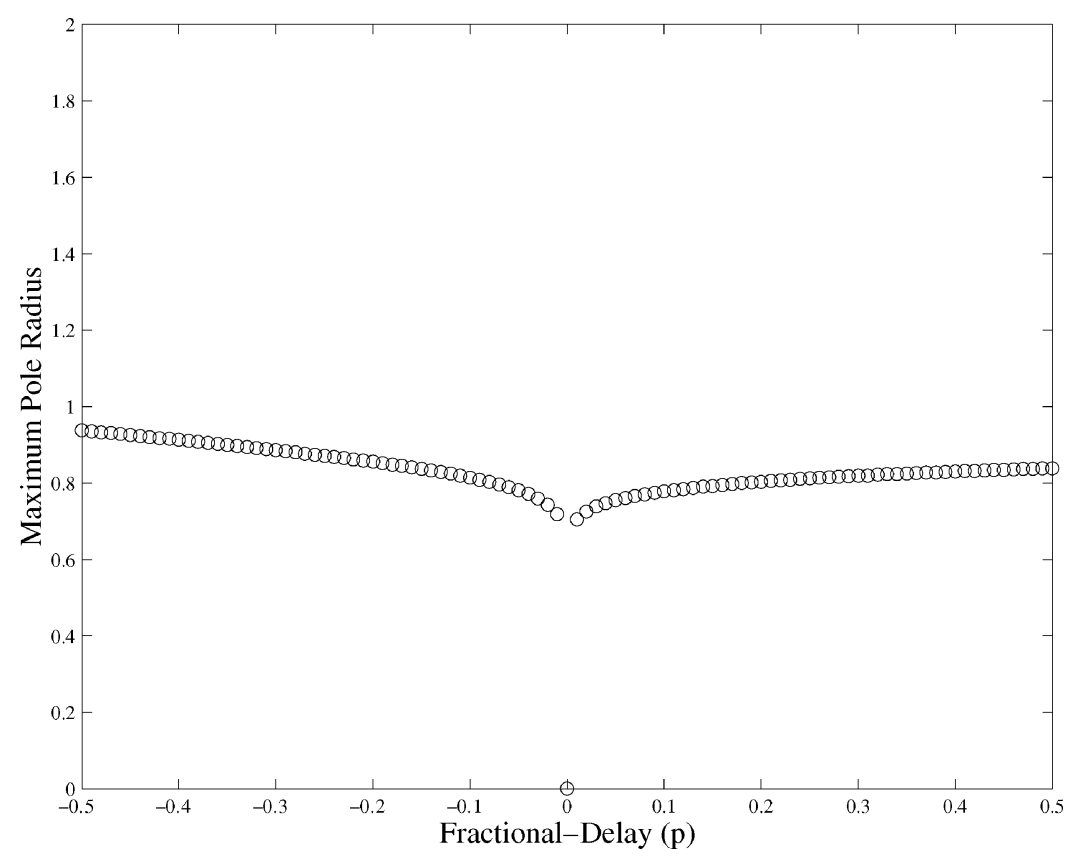

Fig. 7. Maximum pole radius (noniterative WLS design).

4) The LS and WLS design examples have illustrated that the noniterative WLS design can be performed very quickly, and the design results are much better than those using the iterative WLS technique.

Finally, it should also be mentioned that the proposed noniterative WLS technique can be easily generalized to design two-dimensional (2-D) allpass VFD filters by cascading a pair of one-dimensional (1-D) ones [8]. Since the generalization is rather straightforward, we do not detail the design of 2-D allpass VFD digital filters here.

\section{ACKNOWLEDGMENT}

The author would like to thank the reviewers for their constructive comments on the original manuscript.

\section{REFERENCES}

[1] C. W. Farrow, "A continuously variable digital delay element," in Proc. IEEE ISCAS'88, vol. 3, Espoo, Finland, Jun. 6-9, 1988, pp. 2641-2645.

[2] L. Erup, F. M. Gardner, and F. A. Harris, "Interpolation in digital modems-part II: Implementation and performance," IEEE Trans. Commun., vol. 41, pp. 998-1008, Jun. 1993.

[3] S.-C. Pei and C.-C. Tseng, "A comb filter design using fractional-sample delay," in Proc. IEEE ISCAS'97, Hong Kong, Jun. 9-12, 1997, pp. 2228-2231.

[4] — "A comb filter design using fractional-sample delay," IEEE Trans. Circuits Syst. II, Analog Digit. Signal Process., vol. 45, no. 6, pp. 649-653, Jun. 1998.

[5] K. Rajamani, Y.-S. Lai, and C. W. Farrow, "An efficient algorithm for sample rate conversion from CD to DAT," IEEE Signal Proces. Lett., vol. 7, no. 10, pp. 288-290, Oct. 2000.

[6] G.-S. Liu and C.-W. Wei, "A new variable fractional sample delay filter with nonlinear interpolation," IEEE Trans. Circuits Syst. II, Analog Digit. Signal Process., vol. 39, no. 2, pp. 123-126, Feb. 1992.

[7] T.-B. Deng, "High-Resolution Image Interpolation using Two-Dimensional Lagrange-Type Variable Fractional-Delay Filter ," IEICE, Hirosaki, Japan, Tech. Rep. SIS2004-60, 2005.
[8] T.-B. Deng and W.-S. Lu, "Weighted least-squares method for designing variable fractional delay 2-D FIR digital filters," IEEE Trans. Circuits Syst. II, Analog Digit. Signal Process., vol. 47, no. 2, pp. 114-124, Feb. 2000.

[9] T. I. Laakso, V. Valimaki, M. Karjalainen, and U. K. Laine, "Splitting the unit delay: Tools for fractional delay filter design," in IEEE Signal Process. Mag., vol. 13, Jan. 1996, pp. 30-60.

[10] A. Tarczynski, G. D. Cain, E. Hermanowicz, and M. Rojewski, "WLS design of variable frequency response FIR filters," in Proc. IEEE ISCAS'97, Hong Kong, Jun. 9-12, 1997, pp. 2244-2247.

[11] W.-S. Lu and T.-B. Deng, "An improved weighted least-squares design for variable fractional delay FIR filters," IEEE Trans. Circuits Syst. II, Analog Digit. Signal Process., vol. 46, no. 8, pp. 1035-1040, Aug. 1999.

[12] T.-B. Deng, "Discretization-free design of variable fractional-delay FIR digital filters," IEEE Trans. Circuits Syst. II, Analog Digit. Signal Process., vol. 48, no. 6, pp. 637-644, Jun. 2001.

[13] _ _ "Closed-form design and efficient implementation of variable digital filters with simultaneously tunable magnitude and fractional-delay," IEEE Trans. Signal Process., vol. 52, no. 6, pp. 1668-1681, Jun. 2004.

[14] T.-B. Deng and Y. Nakagawa, "SVD-based design and new structures for variable fractional-delay digital filters," IEEE Trans. Signal Process., vol. 52, no. 9, pp. 2513-2527, Sep. 2004.

[15] V. Valimaki and T. I. Laakso, "Principle of fractional delay filters," in Proc. IEEE ICASSP'00, May 2000, pp. 3870-3873.

[16] M. Makundi, T. I. Laakso, and V. Valimaki, "Efficient tunable IIR and allpass structures," Electron. Lett., vol. 37, pp. 344-345, Mar. 2001.

[17] M. Makundi, V. Valimaki, and T. I. Laakso, "Closed-form design of tunable fractional-delay allpass filter structures," in Proc. IEEE ISCAS 2001, vol. IV, Sydney, Australia, May 2001, pp. 434-437.

[18] C.-C. Tseng, "Design of 1-D and 2-D variable fractional delay allpass filters using weighted least-squares method," IEEE Trans. Circuits Syst. I, Fundam. Theroy Appl., vol. 49, no. 10, pp. 1413-1422, Oct. 2002.

[19] J. Y. Kaakinen and T. Saramaki, "An algorithm for the optimization of adjustable fractional-delay all-pass filters," in Proc. IEEE ISCAS'04, vol. III, Vancouver, QC, Canada, May 23-26, 2004, pp. 153-156.

[20] M. Lang and T. I. Laakso, "Simple and robust method for the design of allpass filters using least-squares phase error criterion," IEEE Trans. Circuits Syst. II, Analog Digit. Signal Process., vol. 41, no. 1, pp. 40-48, Jan. 1994.

[21] T. Q. Nguyen, T. I. Laakso, and R. D. Koilpillai, "Eigenfilter approach for the design of allpass filters approximating a given phase response," IEEE Trans. Signal Process., vol. 42, no. 9, pp. 2257-2263, Sep. 1994. 
[22] K. E. Steiglitz and L. E. McBridge, "A technique for the identification of linear systems," IEEE Trans. Autom. Control, vol. AC-10, pp. 461-464, Oct. 1965.

[23] P. A. Regalia, "Comments on 'A weighted least-squares method for the design of stable 1-D and 2-D IIR digital filters'," IEEE Trans. Signal Process., vol. 47, no. 7, pp. 2063-2065, Jul. 1999.

[24] W.-S. Lu, S.-C. Pei, and C.-C. Tseng, "A weighted least-squares method for the design of stable 1-D and 2-D IIR digital filters," IEEE Trans. Signal Process., vol. 46, no. 1, pp. 1-10, Jan. 1998.

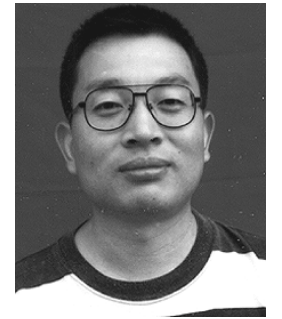

Tian-Bo Deng (M'92-SM'99) received the Ph.D. degree in electronic engineering from Tohoku University, Sendai, Japan, in 1991.

He was with the Department of Information and Computer Sciences, Toyohashi University of Technology, Toyohashi, Japan, as a Research Associate from 1991 to 1992. In 1992, he was selected by the Japanese Government as a Special Researcher for carrying out the Basic-Science-Program at the Institute of Physical and Chemical Research (RIKEN), Wako, Japan. In 1994, he joined the Department of Information Science, Faculty of Science, Toho University, Funabashi, Japan, as an Assistant Professor, and then as an Associate Professor since 1998 From 1998 to 1999, he was also a Visiting Professor with the Department of Electrical and Computer Engineering, University of Victoria, BC, Canada.

His research interests include speech processing, design theory of constant multidimensional digital filters, and design theory of variable one-dimensional and variable multidimensional digital filters. 